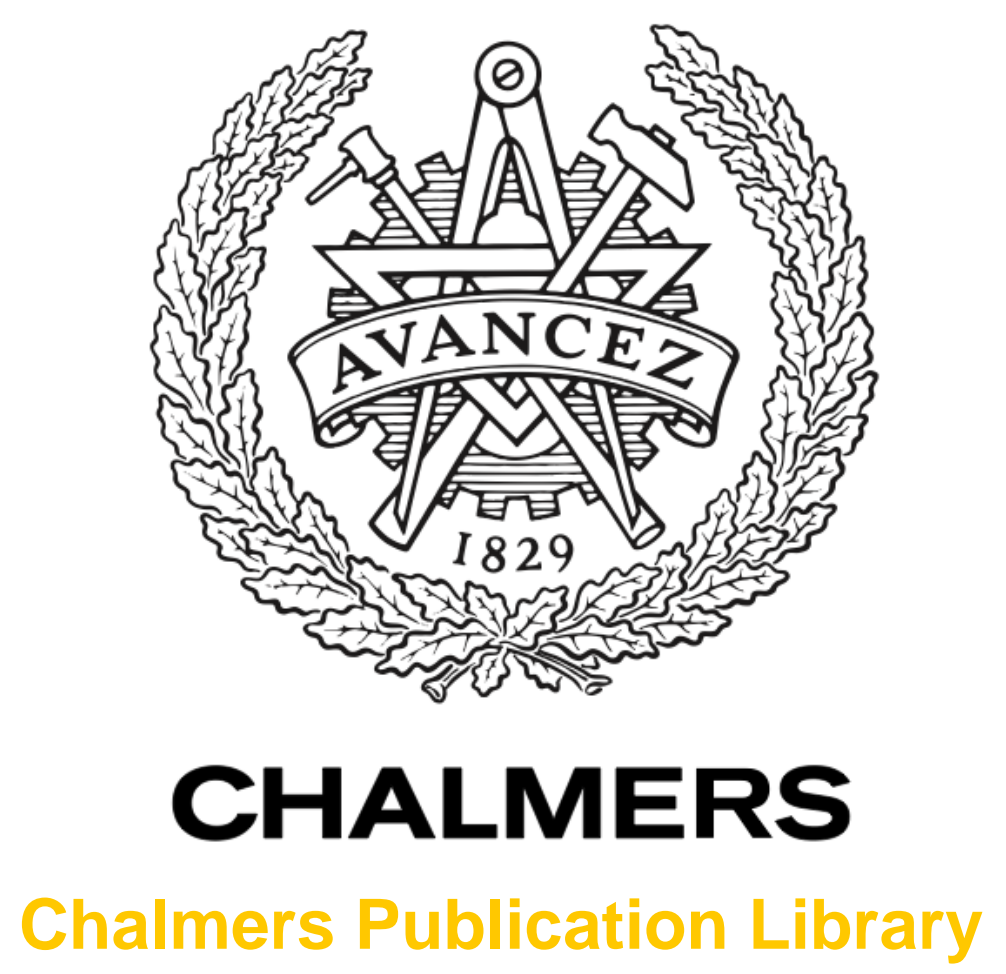

\title{
Achievable rates of multidimensional multisphere distributions
}

This document has been downloaded from Chalmers Publication Library (CPL). It is the author's version of a work that was accepted for publication in:

\section{Optics InfoBase Conference Papers}

Citation for the published paper:

Essiambre, R. ; Karout, J. ; Agrell, E. et al. (2017) "Achievable rates of multidimensional multisphere distributions". Optics InfoBase Conference Papers, vol. Part F40-OFC 2017 pp. Article no 7937455.

http://dx.doi.org/10.1364/OFC.2017.W4A.4

Downloaded from: http://publications.lib.chalmers.se/publication/250919

Notice: Changes introduced as a result of publishing processes such as copy-editing and formatting may not be reflected in this document. For a definitive version of this work, please refer to the published source. Please note that access to the published version might require a subscription. 


\title{
Achievable Rates of Multidimensional Multisphere Distributions
}

\author{
Johnny Karout ${ }^{(1,2,3)}$, René-Jean Essiambre $^{(1)}$, Erik Agrell $^{(2)}$, and Antonia Tulino ${ }^{(1)}$ \\ (1) Nokia - Bell Labs, 791 Holmdel Road, Holmdel, NJ 07733, USA \\ (2) Chalmers University of Technology, SE-412 96 Gothenburg, Sweden \\ ${ }^{(3)}$ Ericsson Research, Ericsson AB, 16480 Stockholm, Sweden \\ johnny.karout@ericsson.com,rene.essiambre@nokia.com
}

\begin{abstract}
The mutual information (MI) of multidimensional multisphere distributions in arbitrary dimensions in the presence of additive white Gaussian noise is derived. We show for instance that 2-D distributions have higher MI than 4-D ones in a range of signal-to-noise ratios.
\end{abstract}

OCIS codes: $060.4080,060.4510,060.0060$.

\section{Introduction}

The choice of modulation formats plays a key role in meeting the demands of future communication systems as it impacts spectral efficiency, power efficiency, and complexity. Constellations in $N$ dimensions $(N$-D) are of interest as they can provide increased spectral and power efficiencies. The dimensions that can be exploited in multidimensional constellations are time, frequency (wavelength), space (fiber cores or fiber modes) and polarizations. For channels where the noise has rotationally invariant statistics, the study of rotationally invariant constellations is of great interest. The multidimensional Gaussian distribution is the most common such distribution, and it is capacity-achieving over the additive white Gaussian noise (AWGN) channel under an average power constraint [1]. However, more practical input distributions assume being discrete in amplitude, but still keeping rotational invariance, i.e., continuous and uniform in phase (where "phase" may be interpreted in a multidimensional sense). Such distributions are called multisphere distributions in $N$-D (or multiring distributions in 2-D) and are of great interest for developing practical constellations.

Several papers have considered the properties of multiring distributions for a wide range of channels under average and/or peak power constraints for AWGN [2-4] as well as for the nonlinear fiber channel [5-8]. In this paper, we present a low-complexity analytical expression for calculating the mutual information of an $N$-D multisphere distribution of arbitrary $N$ for the AWGN channel [9]. We present the information rate of various multidimensional multisphere distributions and show a case where 2- $D$ multiring constellations can exceed the corresponding 4- $D$ distribution.

\section{System Model and Mutual Information}

We consider a discrete-time AWGN channel $\mathbf{Y}=\mathbf{X}+\mathbf{N}$, where $\mathbf{X}$ is an $N$-D real input vector and $\mathbf{N}$ is an $N$-D normally distributed noise vector with mean 0 and variance $\sigma^{2}=N_{0} / 2$ per real dimension.

The mutual information between input and output is given by $I(\mathbf{X} ; \mathbf{Y})=h(\mathbf{Y})-h(\mathbf{Y} \mid \mathbf{X})$ [1], where the differential entropies are

$$
h(\mathbf{Y})=-\int f_{\mathbf{Y}}(\mathbf{y}) \log _{2} f_{\mathbf{Y}}(\mathbf{y}) \mathrm{d} \mathbf{y} \quad \text { and } \quad h(\mathbf{Y} \mid \mathbf{X})=h(\mathbf{N})=\frac{N}{2} \log _{2} 2 \pi e \sigma^{2} .
$$

The maximum mutual information under an average power constraint is the AWGN channel capacity $C=$ $N / 2 \log _{2}(1+2 \mathrm{SNR} / N)$, where SNR $=\mathbb{E}\left[\|\mathbf{X}\|^{2}\right] / N_{0}$ with $\|\cdot\|$ denoting the norm. We furthermore define the SNR per 4 dimensions as $4-\mathrm{D}$ SNR $=(4 / N)$ SNR.

We consider the case where the input vector $\mathbf{X}$ is distributed according to an $N$-D multisphere distribution (e.g., see Fig. 1), where the probabilities and radii of each hypersphere labeled from $k=1, \ldots, K$ are $p_{k}$ and $s_{k}$, respectively. Under this assumption, and by denoting with $f_{\|\mathbf{X}\|}$ the distribution of the magnitude of input vector $\mathbf{X}$, the mutual information between $\mathbf{X}$ and $\mathbf{Y}$ in bits per $N$ dimensions is given by [9]

$$
I(\mathbf{X} ; \mathbf{Y})=-\int_{0}^{\infty} f_{\tilde{R}}(\tilde{r}) \log _{2} \frac{f_{\tilde{R}}(\tilde{r})}{\tilde{r}^{N-1}} \mathrm{~d} \tilde{r}+\log _{2} \frac{2}{\Gamma(N / 2)}-\frac{N}{2} \log _{2} 2 e
$$




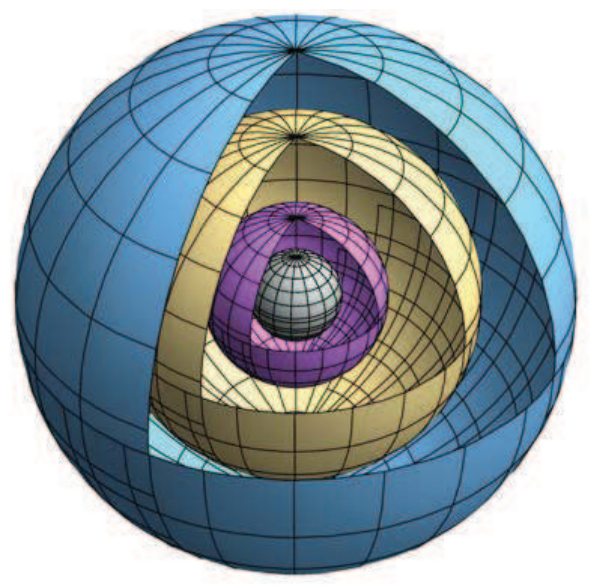

Fig. 1: A visual representation of a 3-D multisphere distribution composed of four hyperspheres.

where

$$
f_{\tilde{R}}(\tilde{r})=\sum_{k=1}^{K} p_{k} \chi\left(\tilde{r}, \frac{s_{k}}{\sigma}\right), \quad \chi(\tilde{r}, \tilde{s})=\frac{\tilde{r}^{N / 2}}{\tilde{s}^{N / 2-1}} \exp \left(-\frac{\tilde{r}^{2}+\tilde{s}^{2}}{2}\right) I_{N / 2-1}(\tilde{r} \tilde{s}),
$$

and $I_{v}(u)$ is the $v$ th order modified Bessel function of the first kind. Note that the $N$-dimensional integral of $h(\mathbf{Y})$ in Eq. (1) has been reduced to a single integral in Eq. (2), enabling fast computation of the maximum information rate, or constrained capacity, of multisphere distributions. This represents a considerable reduction in complexity and fast evaluation of how much information these constellations can carry. For the case where $\mathbf{X}$ is distributed according to an $N$-D multisphere distribution with $K$ uniformly spaced hyperspheres and equal probabilities per hypersphere, then

$$
f_{\tilde{R}}(\tilde{r})=\frac{1}{K} \sum_{k=1}^{K} \chi(\tilde{r}, k \Delta) \quad \text { where } \quad \Delta=\sqrt{\frac{12 \mathrm{SNR}}{2 K^{2}+3 K+1}} .
$$

As a further special case, setting $K=1$ and $N=2$ in Eqs. (2) and (3) gives the constrained capacity of constantamplitude constellations, i.e., purely phase-modulated transmission schemes, in the presence of AWGN (e.g., see [10]).

\section{Mutual Information of Multisphere Distributions}

We evaluate the mutual information by substituting Eqs. (3) and (4) into (2) and by numerically evaluating the resulting expression for a uniform distribution of $K N$-D hyperspheres. Note that transmission of a sequence of symbols in time in single-mode fibers uses 4-D (4 real dimensions) for each symbol. Figure 2 shows the mutual information as a function of the 4-D SNR, the ratio between energy per four dimensions and $N_{0}$, for a 4-D signal composed of two independent 2-D $(N=2)$ signals (e.g., one in each polarization state) and a single 4-D $(N=4)$ signal (e.g. a symbol using both polarizations) for a different number of hyperspheres $K=\{1,4,16\}$. An increased number of hyperspheres allows approaching the AWGN capacity more closely since they fill up the space more uniformly. It is interesting to note that for $K=16$ the mutual information of two 2-D constellations exceeds the case of a single 4-D constellation for an 4-D SNR up to $\sim 35 \mathrm{~dB}$ (see inset of Fig. 2). This is a range of relevance to fiber capacity estimation [6].

Multidimensional single-hypersphere distributions are also of interest as they provide potential advantages in nonlinear transmission [11]. Figure 3 shows the mutual information of a single hypersphere up to 32 (real) dimensions normalized to 4-D. The Gaussian 4-D distribution is shown as a reference. In one dimension, a sphere is represented by 2 points (binary) and the mutual information saturates to 4 bits/symbol at high 4-D SNR. As the dimensionality of the hypersphere increases, the AWGN capacity is approached very closely for 4-D SNR in the range of $\sim 10-30$ $\mathrm{dB}$ with a relatively low dimensionality. The case of a 4-D distribution (two polarizations) for different numbers of hyperspheres is shown in Fig. 4. It is interesting to note that increasing the number of hyperspheres allows getting closer to the AWGN capacity up to $\sim 30-\mathrm{dB} 4-\mathrm{D}$ SNR but at a relatively slow rate (see inset of Fig. 4). This suggests 


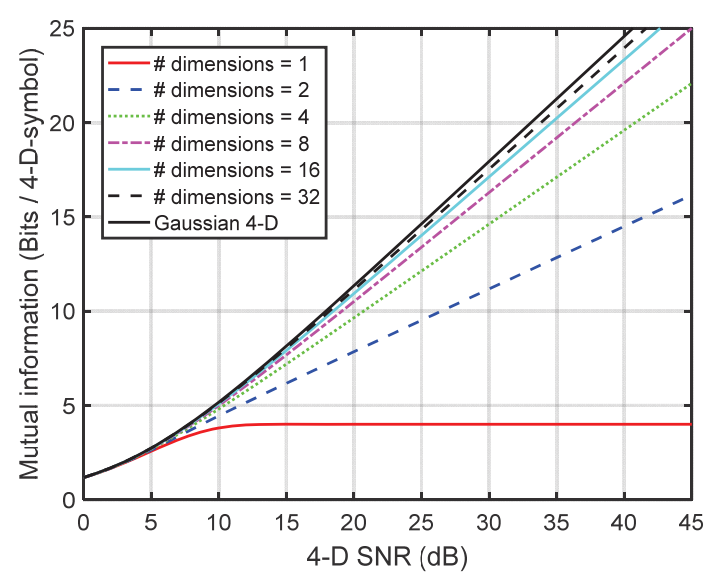

Fig. 3: Mutual information versus 4-D SNR of a hypersphere, $K=1$, with dimensions $N=\{1,2,4,8,16,32\}$.

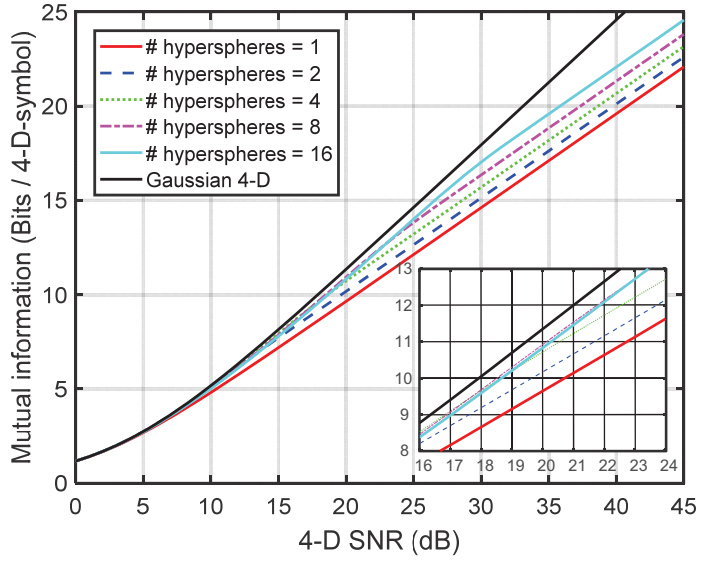

Fig. 4: Mutual information versus 4-D SNR for various numbers of 4-D, $N=4$, hyperspheres, with $K=$ $\{1,2,4,8,16\}$.

that increasing the number of dimensions of a distribution may be a more attractive way to approach capacity than the number of hyperspheres.

\section{Conclusions}

A simplified expression allowing for the fast computation of the mutual information of an $N$-D multisphere distribution is derived in the presence of additive white Gaussian noise, by exploiting the rotationally invariant properties of the noise and the distribution itself. Results of constrained capacities for different multisphere distributions are presented giving insights for the design of highly efficient modulation formats, in particular of 2-D versus 4-D constellations.

\section{Acknowledgments}

This work was supported by the Swedish Foundation for Strategic Research under Grant RE07-0026, the Ericsson Research Foundation, the Friends of Chalmers Foundation, and the Royal Society of Arts and Sciences in Gothenburg. The authors would like to thank G. J. Foschini and R. W. Tkach for their support.

\section{References}

1. C. E. Shannon, “A mathematical theory of communication," Bell Syst. Tech. J., vol. 27, pp. 379-423 and 623656, 1948.

2. S. Shamai and I. Bar-David, "The capacity of average and peak-power-limited quadrature Gaussian channels," IEEE Trans. Inf. Theory, vol. 41, no. 4, pp. 1060-1071, July 1995.

3. T. H. Chan, S. Hranilovic, and F. R. Kschischang, "Capacity-achieving probability measure for conditionally Gaussian channels with bounded inputs," IEEE Trans. Inf. Theory, vol. 51, no. 6, pp. 2073-2088, June 2005.

4. M. C. Gursoy, H. V. Poor, and S. Verdú, "The noncoherent Rician fading channel—Part I: Structure of the capacity-achieving input," IEEE Trans. Wireless Commun., vol. 4, no. 5, pp. 2193-2206, Sept. 2005.

5. T. Freckmann, R.-J. Essiambre, P. J. Winzer, G. J. Foschini, and G. Kramer, "Fiber capacity limits with optimized ring constellations," IEEE Photon. Technol. Lett., vol. 21, no. 20, pp. 1496-1498, Oct. 2009.

6. R.-J. Essiambre, G. Kramer, P. J. Winzer, G. J. Foschini, and B. Goebel, "Capacity limits of optical fiber networks," J. Lightw. Technol., vol. 28, no. 4, pp. 662-701, Feb. 2010.

7. H. Ghozlan and G. Kramer, "Interference focusing for simplified optical fiber models with dispersion," in Proc. IEEE Int. Symp. Inf. Theory, Saint Petersburg, Russia, July-Aug. 2011, pp. 376-379.

8. E. Agrell, "Nonlinear fiber capacity," in Proc. Eur. Conf. Opt. Commun. (ECOC), London, UK, Sept. 2013.

9. J. Karout, R.-J. Essiambre, E. Agrell, and A. Tulino, "Achievable rates of multidimensional rotationally invariant distributions," arXiv preprint arXiv:1604.04256, 2016.

10. A. D. Wyner, “Bounds on communication with polyphase coding,” Bell Syst. Tech. J., pp. 523-559, Apr. 1966.

11. R.-J. Essiambre, G. J. Foschini, G. Kramer, and P. J. Winzer, "Capacity limits of information transport in fiberoptic networks," Phys. Rev. Lett., vol. 101, 2008, paper 163901. 